\title{
Comparison of microRNA expression profiles in K562-cells-derived microvesicles and parental cells, and analysis of their roles in leukemia
}

\author{
XIAOMEI CHEN ${ }^{1 *}$, WEI XIONG ${ }^{2 *}$ and HUIYU LI ${ }^{3}$ \\ ${ }^{1}$ Center for Biotherapy, Gansu Provincial Hospital, Lanzhou, Gansu $730000 ;{ }^{2}$ Center of Clinical Laboratory, \\ The Second Affiliated Hospital of Zhejiang University School of Medicine at Binjiang, Hangzhou, Zhejiang 310009; \\ ${ }^{3}$ Center for Stem Cell Research and Application, Institute of Hematology, Union Hospital, \\ Tongji Medical College, Huazhong University of Science and Technology, Wuhan, Hubei 430022, P.R. China
}

Received April 16, 2015; Accepted July 20, 2016

DOI: $10.3892 / \mathrm{ol} .2016 .5308$

\begin{abstract}
Microvesicles (MVs) are 30-1,000-nm extracellular vesicles that are released from a multitude of cell types and perform diverse cellular functions, including intercellular communication, antigen presentation, and transfer of proteins, messenger RNA and microRNA (also known as miR). MicroRNAs have been demonstrated to be aberrantly expressed in leukemia, and the overall microRNA expression profile may differentiate normal blood cells vs. leukemia cells. MVs containing microRNAs may enable intercellular cross-talk in vivo. This prompted us to investigate specific variations of microRNA expression patterns in MVs derived from leukemia cells. The present study examined the microRNA expression profile of MVs from chronic myeloid leukemia K562 cells and that of MVs from normal human volunteers' peripheral blood cells. The potential targets of the differentially expressed microRNAs were predicted using computational searches. Bioinformatic analyses of the predicted target genes were performed for further evaluation. The present study analyzed microRNAs of MVs derived from leukemia and normal cells, and characterized specific microRNAs expression. The results revealed that MVs derived from K562 cells expressed 181 microRNAs of the 888 microRNAs assessed. Further analysis revealed that 16 microRNAs were downregulated, while 7 were upregulated in these MVs. In addition, significant
\end{abstract}

Correspondence to: Professor Huiyu Li, Center for Stem Cell Research and Application, Institute of Hematology, Union Hospital, Tongji Medical College, Huazhong University of Science and Technology, 1277 Jiefang Road, Qiaokou, Wuhan, Hubei 430022, P.R. China

E-mail: huiyuli@163.com

*Contributed equally

Key words: microRNAs, microvesicles, leukemia, target, pathway, chromosome location differences in microRNA expression profiles between MVs derived from K562 cells and K562 cells were identified. The present results revealed that 77 and 122 microRNAs were only expressed in MVs derived from K562 cells and in K562 cells, respectively. There were 104 microRNAs co-expressed in MVs derived from K562 cells and in K562 cells. Target gene-related pathway analyses demonstrated that the majority of the dysregulated microRNAs were involved in pathways associated with leukemia, particularly the mitogen-activated protein kinase (MAPK) and the p53 signaling pathways. By further conducting microRNA gene network analysis, the present study revealed that the $\mathrm{miR}-15 \mathrm{a} / \mathrm{b}$, miR-16, $\mathrm{miR}-17$ and miR-30 families were likely to play a role in the regulation of the MAPK signaling pathway. Since K562 cells presented the $t(9 ; 22)$ translocation, the current study further examined the predicted function of 12 microRNAs located in chromosomes 9 [Homo sapiens (hsa)-let-7a, hsa-let-7f, miR-126, miR-126*, miR-23b, miR-24, miR-27b and miR-7] and 22 (hsa-let-7b, miR-1249, miR-130b and miR-185), which were expressed both in MVs derived from K562 cells and in K562 cells. The present study identified microRNAs of MVs from leukemia and normal cells, and characterized the expression of specific microRNAs. The current study is also the first to identify and characterize distinct microRNA expression between MVs derived from K562 cells and K562 cells. These findings highlight that a number of microRNAs from leukemia-derived MVs may contribute to the development of hematopoietic malignancies. Further investigation may reveal the function of these differentially expressed microRNAs and may provide potential targets for novel therapeutic strategies.

\section{Introduction}

Chronic myeloid leukemia (CML) refers to a group of neoplasias that are defined by a unique genetic aberration, the breakpoint cluster region-Abelson murine leukemia viral oncogene homolog 1 (BCR-ABL1) fusion gene (1). CML is also reognized as one of the best examples for molecular targeted therapy (2). However, numerous aspects of the pathogenesis of CML have not been elucidated thus far, including the mechanisms of blastic 
crises, the causes of genetic instabilities such as the inactivation of tumor suppressor genes, and the oncogenic signaling pathways downstream of the BCR-ABL1 fusion gene product (3).

In recent years, microvesicles (MVs) in the leukemia microenvironment have been drawn researchers' attention $(4,5)$. MVs, also known as exosomes or microparticles, are vesicles comprised of various cell organelles of diameters ranging from 30 to $1,000 \mathrm{~nm}$ (6). The formation of MVs can happen on the cellular membrane surface or the endosome (7). The functions of MVs are closely associated with the contents in the vesicle (8). Despite their small size, MVs are enriched in bioactive molecule, including growth factors and their receptors, proteinases, adhesion molecules, signal molecules, DNA, messenger (m)RNA and microRNA (also known as miR) (4). These bioactive molecules collectively function as the signaling complex (9-12). It has been reported that MVs released from CML cells are important in angiogenesis $(13,14)$ and immunosuppression $(15)$. The mechanisms of these MVs' roles have not been yet elucidated. Previous studies revealed that genetic exchanges of microRNAs between cells can be accomplished through MVs (16-18). MVs can fuse with the cellular membrane and transfer all the aforementioned bioactive molecules to receptor cells (17), thus being important in cell-to-cell communication.

MicroRNAs are non-coding, single-stranded RNAs of 21-25 nucleotides, which have recently been implicated in the regulation of a number of biological processes, including development, differentiation, apoptosis, proliferation and hematopoiesis (19). MicroRNAs regulate gene expression by promoting the degradation of their target mRNA or repressing its translation (19). MicroRNA expression is tissue-specific, and has been demonstrated to be altered in a number of human cancers $(20,21)$. In CML, abnormal expression of several microRNAs has been described, including miR-15a, miR-16, miR-142, miR-155, miR-181, miR-221, let7a and the polycistronic miR-17-92 cluster (22-24).

Notably, a connection between MVs and microRNA has been recently proposed (25). Specific miRNAs regulate hematopoietic cell differentiation and development (26). Previous studies have indicated that microRNA packaged within MVs may be transported extracellularly $(25,27-30)$. It is possible that these microRNAs in MVs reflect the miRNA signature of the parental tumor (31). Employing MVs to transfer genetic material would be an efficient transfer method between cells, and MVs containing microRNAs would enable intercellular and inter-organ communication in the body.

The present study explored the hypothesis that microRNAs are contained within MVs derived from the CML cell line K562. The microRNA expression profile of MVs from K562 cells and from normal human volunteers' peripheral blood cells was examined, and the microRNA expression in K562 cells-derived MVs was compared with that in K562 cells, and their roles in leukemia were analyzed. The objectives of the present study were to determine whether the microRNAs contained within leukemia-cell-derived MVs mirrored those of leukemia cells and thus, could be used diagnostically and therapeutically.

\section{Materials and methods}

Cell line and control samples. The K562 cells (kindly provided by Dr.Jingqiong $\mathrm{Hu}$, Tongji Medical College,
Huazhong University of Science and Technology, Wuhan, China) were grown in RPMI 1640 medium (Gibco; Thermo Fisher Scientific, Inc., Waltham, MA, USA) supplemented with 10\% MV-free (by ultrafiltration) fetal bovine serum (Gibco; Thermo Fisher Scientific, Inc.), 25 mM 4-(2-hydroxyethyl)1-piperazineethanesulfonic acid, $24 \mathrm{mM}$ sodium bicarbonate, $2 \mathrm{mM}$ L-glutamine, $100 \mathrm{IU} / \mathrm{ml}$ penicillin and $100 \mathrm{IU} / \mathrm{ml}$ streptomycin in a humidified $5 \% \mathrm{CO}_{2}$ atmosphere at $37^{\circ} \mathrm{C}$. Cell viability was evaluated by trypan blue exclusion assay, and all cultures utilized were $95 \%$ viable. A method was established using ultrafiltration to remove MVs from fetal bovein serum. Initially, debris in fetal bovein serum was removed by $0.22 \mu \mathrm{m}$ filter (EMD Millipore, Billerica, MA, USA). Subsequently, the supernatant was purified by stirring ultrafiltration with a 100,000 molecular weight cut off ultrafiltration membrane (EMD Millipore) to remove MVs.

Peripheral blood was collected in ethylenediaminetetraacetic acid-coated tubes from healthy donors at Union Hospital (Tongji Medical College, Huazhong University of Science and Technology) between August and October 2012. The criteria for volunteers selection consisted of no recent illnesses or treatments for a chronic medical condition. No medical history was obtained from the donors. The collection of blood occurred between the morning and early afternoon. The average age for the female and male donors was 35.79 and 31.81 years, respectively. Written informed consent was obtained from all volunteers and this study was approved by the ethics committee of Tongji Medical College, Huazhong University of Science and Technology.

Isolation of MVs from cell culture supernatants. Supernatants were collected from cell culture after $24 \mathrm{~h}$. Cells were centrifuged at $300 \mathrm{x}$ g for $30 \mathrm{~min}$, and the supernatant was used for MVs preparation. Cell debris was removed by centrifugation at 2,500 x g for $30 \mathrm{~min}$. MVs were purified by ultracentrifugation at $16,000 \mathrm{x}$ g for $120 \mathrm{~min}$, and subsequently washed with sterile filtered phosphate-buffered saline (PBS). The samples were resuspended in radioimmunoprecipitation assay (RIPA) buffer supplemented with a protease inhibitor cocktail (Agilent Technologies, Inc., Santa Clara, CA, USA) and stored at $-80^{\circ} \mathrm{C}$. All centrifugations were accomplished at $4^{\circ} \mathrm{C}$. MVs quantity was determined with the Pierce BCA Protein Assay kit (Thermo Fisher Scientific, Inc., Waltham, MA, USA).

Isolation of MVs from normal human volunteers' peripheral blood cells. Peripheral blood was centrifuged at $400 \mathrm{x}$ g for $30 \mathrm{~min}$, and the plasma was used for MVs preparation. Cell debris and platelets were removed by centrifugation at $2,500 \mathrm{~g}$ for $20 \mathrm{~min}$ twice. MVs were purified by ultracentrifugation at $16,000 \mathrm{x}$ g for $120 \mathrm{~min}$, and subsequently washed with sterile filtered PBS (32). All centrifugations were accomplished at $4^{\circ} \mathrm{C}$. MV pellets were resuspended in RIPA buffer supplemented with a protease inhibitor cocktail (Agilent Technologies, Inc.) and stored at $-80^{\circ} \mathrm{C}$. MVs quantity was determined with the Pierce BCA Protein Assay kit (Thermo Fisher Scientific, Inc.).

Transmission electron microscopy. For transmission electron microscopy, the pelleted MVs were fixed in $2.5 \%$ $(\mathrm{w} / \mathrm{v})$ glutaraldehyde in PBS, dehydrated and embedded in Epon (SPI Supplies, Inc., West Chester, PA, USA). Ultrathin 
Table I. Expression signatures of dysregulated miRNAs from K562-derived MVs.

A, Upregulated microRNAs in K562-derived MVs

\begin{tabular}{lcc}
\hline Name & P-value & Fold-change \\
\hline miR-151-3p & 0.009770351 & 0.0134710 \\
miR-1974_v14.0 & 0.003311413 & 0.0146479 \\
miR-26a & 0.003024278 & 0.0146984 \\
miR-223 & 0.049822803 & 0.0147095 \\
miR-23b & 0.024271710 & 0.0390509 \\
miR-103 & 0.023439787 & 0.0406894 \\
miR-24 & 0.003045386 & 0.0496698 \\
miR-361-5p & 0.036743575 & 0.0531059 \\
miR-21 & 0.024356252 & 0.0796379 \\
miR-22 & 0.007922998 & 0.0820633 \\
miR-126* & 0.022427225 & 0.0843179 \\
miR-107 & 0.048353604 & 0.1005024 \\
miR-27b & 0.033929142 & 0.1036153 \\
miR-93 & 0.000452252 & 0.1485404 \\
miR-27a & 0.021547485 & 0.1844731 \\
miR-185 & 0.039805662 & 0.4350724 \\
\hline
\end{tabular}

B, Downregulated microRNAs in K562-derived MVs

\begin{tabular}{lcr}
\hline Name & P-value & Fold-change \\
\hline miR-494 & 0.033709016 & 7.1837779 \\
miR-1275 & 0.040862084 & 11.0616334 \\
miR-483-5p & 0.030694947 & 12.8443913 \\
miR-1308_v15.0 & 0.015232616 & 26.8287621 \\
miR-575 & 0.015022667 & 46.2936787 \\
miR-1268 & 0.015112414 & 59.5764594 \\
miR-125a-3p & 0.027056158 & 96.4917155 \\
\hline
\end{tabular}

miR, microRNA; MV, microvesicle.

sections (65-nm) were cut and stained with uranyl acetate (SPI Supplies, Inc.) and Reynold's lead citrate (SPI Supplies, Inc.). The sections were examined in a Tecnai G2 12 transmission electron microscope (FEI, Hillsboro, OR, USA).

RNA extraction and purification. Total RNA was isolated from K562 cells and MVs using the mirVana microRNA Isolation kit according to the manufacturer's protocol (Ambion; Thermo Fisher Scientific, Inc.). The quality, yield and size of the microRNA fractions were analyzed using a 2100 Bioanalyzer (Agilent Technologies, Inc.). For RNA isolated from mononuclear cells, only an RNA integrity number $(\mathrm{RIN}) \geq 7$ was used, along with its matched plasma sample for profiling. Since the intact 18 and 28s ribosomal RNA were variable in the MVs, the RIN was not a constraint for these samples, although a RIN between 6.5 and 6.8 was observed.
MicroRNA profiling. RNA from K562 cells, and MVs both from K562 cells and normal human volunteers' peripheral blood cells were used for microRNA microarray. Human microRNA microarrays from Agilent Technologies, Inc., which contain probes for 888 human microRNAs from the miRBase v14.0 (mirbase.org/pub/mirbase/14.0/), were used in the study. In total, $100 \mathrm{ng}$ of total RNA extracted from serum was used as inputs for sample labeling and hybridization preparation, following the manufacturer's protocol.

The microarray image information was converted into spot intensity values using Scan Control Software version 7.0 (Agilent Technologies, Inc.). The signal upon background subtraction was exported directly into the GeneSpring GX 11.0 software (Agilent Technologies, Inc.) for quartile normalization and further analysis.

Validation of microarray data. For testing of candidate microRNAs acquired on microarrays, reverse transcriptionquantitative polymerase chain reaction (RT-qPCR) was performed using miScript SYBR Green PCR kit (Qiagen $\mathrm{GmbH}$, Hilden, Germany). The assays were performed on three samples for six candidates (miR-494, miR-483-5p, miR-26a, miR-223, miR-21 and miR-22).

Statistical analysis. Data were analyzed using the statistical software package SAS v3.0. (Shanghai Biotechnologies Corporation, Shanghai, China). The statistical significance was calculated by the Student's $t$ test. $\mathrm{P} \leq 0.05$ was considered to indicate a statistically significant difference. Three-way Venn diagrams were used to indicate the number of significant microRNAs $[\mathrm{P}<0.05$, fold-change $(\mathrm{FC})>2$ and $\mathrm{FC}<0.5]$ identified by the Student's $t$ test (K562-MVs vs. control MVs).

Pathway analysis and prediction. The human TargetScan Release 5.1 (http://www.targetscan.org) was used for prediction of microRNA targets. In addition, potential target gene-associated pathways were analyzed using TargetScan Release 5.1 based on the Kyoto Encyclopedia of Genes and Genomes (KEGG) pathway database (http://www.genome. $\mathrm{jp} / \mathrm{kegg} /$ ). The enrichment P-value of the target genes involved in every pathway was calculated, and the regulatory interactions between genes and microRNAs were integrated.

\section{Results}

Transmission electron microscopy. MVs derived from K562 cells and normal human volunteers' peripheral blood cells were collected and observed under a transmission electron microscope, which revealed vesicular structures characteristic of MVs (Fig. 1A and B).

MicroRNA expression profile in K562-derived MVs. A microarray containing probes for 888 human microRNAs was initially used to screen the significant differential expression levels of microRNAs between K562-MVs and control groups (Table I). The filtered and normalized data were subjected to hierarchical cluster analysis comparing the microRNA expression profile of K562-MVs and control group samples. Fig. 2 illustrates the hierarchical clustering of the differentially expressed microRNAs in the pairwise comparison of 
A

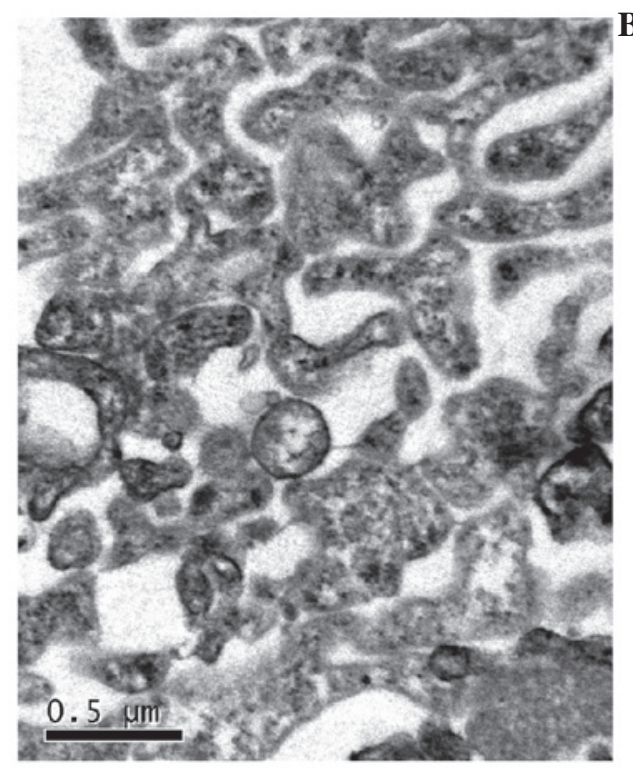

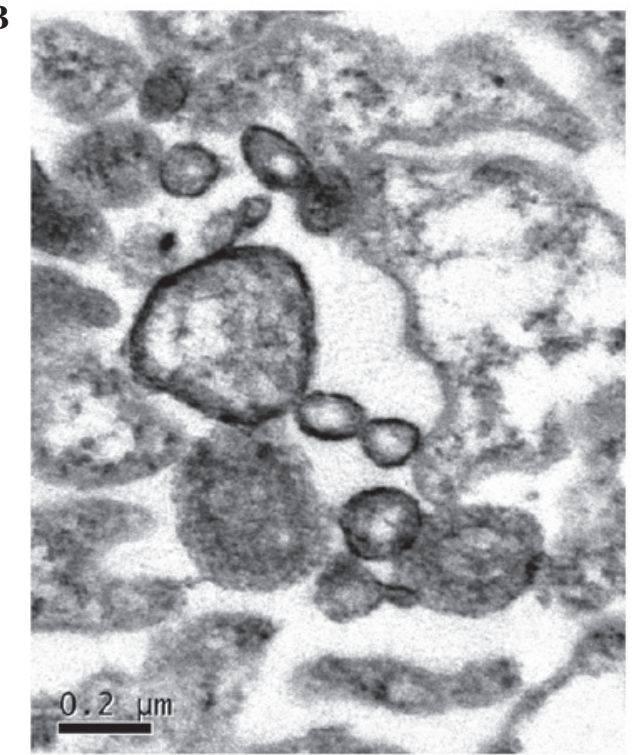

Figure 1. Characterization of MVs. Representative micrographs of transmission electron microscopy of (A) K562 MVs and (B) control MVs exhibiting a spheroid shape. Both types of MVs displayed the same morphology and size. MV, microvesicle.
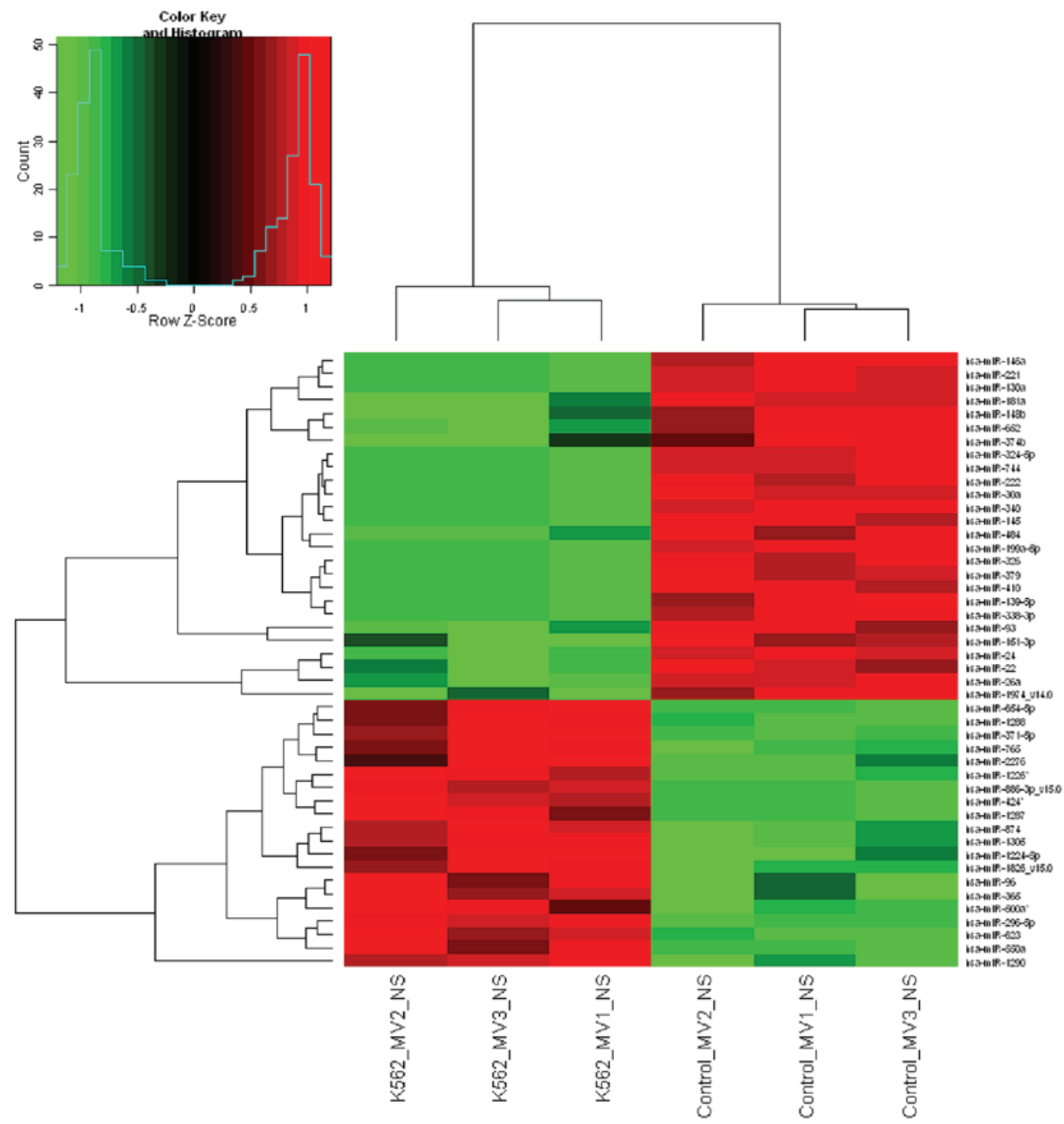

Figure 2. MicroRNA expression of MVs derived from K562 cells. Hierarchical cluster analysis for MVs derived from K562 cells and from normal human volunteers' peripheral blood cells is represented based on filtering criteria. hsa, Homo apiens; miR, microRNA; MV, microvesicle; NS, normalized signal. 
Table II. Expression levels of microRNAs in K562 cells-derived MVs compared with those of microRNAs isolated from K562 cells.

Elevated in cells

miR-142-5p, miR-374a, miR-590-5p,

miR-101, miR-29b, miR-20a*, miR-377,

miR-144, miR-381, miR-374b, miR-424,

miR-21*, miR-140-5p, miR-301a,

miR-19b-1*, miR-29c, miR-342-3p,

miR-486-3p, miR-135b, miR-10a,

miR-154*, miR-301b, miR-127-3p,

miR-379, miR-487b, miR-140-3p,

miR-136, miR-654-3p, miR-299-3p,

miR-331-3p, miR-431*, miR-33a,

miR-410, miR-218, miR-660, miR-148b,

miR-212, miR-223*, miR-100, miR-148a, miR-409-3p, miR-30e*, miR-495,

miR-99b, miR-192, miR-337-5p, miR-186, miR-32, miR-493*, miR-299-5p, miR-376b, miR-183 and miR-324-5p

Equal in cells and MVs

Elevated in MVs

let-7a, let-7b, let-7c, let-7e,

let-7f, hsa-let-7f-1*, let-7g,

let-7i, miR-103, miR-106b,

miR-107, miR-1202,

miR-125a-3p, miR-125a-5p,

miR-125b, miR-126,

miR-1268, miR-1275,

miR-1305, miR-130b,

miR-142-3p, miR-151-3p,

miR-151-5p, miR-15a,

miR-15b, miR-16, miR-17,

miR-17*, miR-185,

miR-19a, miR-19b,

miR-20a, miR-20b, miR-21,

miR-210, miR-22,

miR-223, miR-224,

miR-23a, miR-23b, miR-24, miR-25, miR-26a, miR-26b, miR-27a, miR-27b, miR-29a, miR-30b, miR-30c, miR-30e, miR-494, miR-575, miR-638, miR-7, miR-92a and miR-93
miR-630, miR-671-5p, miR-874, miR-188-5p, miR-483-5p, miR-513a-5p, miR-1224-5p, miR-765, miR-654-5p, miR-135a*, miR-1226*, miR-892b, miR-1183, miR-2276, miR-584, miR-371-5p, miR-500a, miR-150*, miR-422a, miR-520b, miR-155, miR-501-5p, miR-921, miR-628-3p, miR-1287, miR-887, miR-1471, miR-520e, miR-149*, miR-500a*, miR-718, miR-1181, miR-513c, miR-542-5p, miR-564, miR-1299, miR-662, miR-622, miR-490-5p, miR-557, miR-877, miR-602, miR-610, miR-760, miR-125b-1* , miR-202, miR-502-3p, miR-99b*, miR-663, miR-583 and $\mathrm{miR}-617$
K562-MVs K562-ce11s

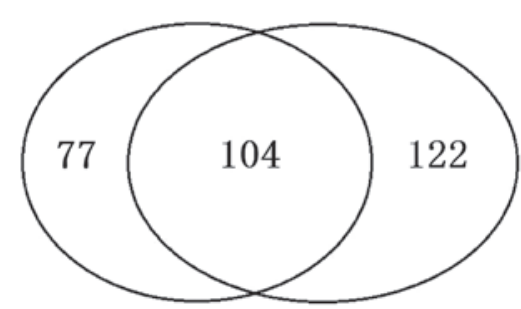

Figure 3. Number and overlap of microRNAs in K562-derived MVs and in their parental cells. MV, microvesicle.

the two samples. There were seven microRNAs, including miR-494, miR-1275, miR-484-5p, miR-1308-v15.0, miR-575, miR-1268 and miR-125a-3p, with significantly higher expression levels in the K562-MV group than in the healthy group ( $\mathrm{FC}=7.18-96.49 ; \mathrm{P}<0.05$; Table I). By contrast, miR-151-3p, miR-1974-v14.0, miR-26a, miR-24, miR-22, miR-93, miR-223, miR-23b, miR-103, miR-361-5p, miR-21, miR-126*, miR-107, miR-27b, miR-27a and miR-185 displayed a significantly lower expression level in the K562-MV group than in the healthy group (FC<0.5; $\mathrm{P}<0.05$; Table I).
Comparison of K562-MVs' microRNA expression with that of parental cells. The presence and levels of specific microRNAs from both K562-MVs and their parental cells were determined using microarray analysis probing for 888 human microRNAs. The microRNA profiles of K562 cells confirmed the alterations previously reported (33). Furthermore, the results demonstrated that 30 microRNAs were above the normalized threshold in the 888 microRNAs, which was calculated based on the 95 percentile of the negative control probe signal in both normal cells and MVs (Fig. 3 and Table II). Of the 303 positive microRNAs, 104 were not significantly different between MVs and their parental K562 cells. By comparison, 77 microRNAs were present at elevated levels within MVs, while 122 were present at a higher proportion in the parental cells .This observation may suggest that the compartmentalization of microRNAs from cells into MVs is an active (selective) process, at least for certain microRNAs.

Validation of microarray data. Two upregulated (miR-494 and miR-484-5p) and four downregulated (miR-26a, miR-22, miR-223 and miR-21) microRNAs of K562-MVs (in terms of their expression levels compared with those in the control) were selected for microarray data validation via RT-qPCR, 
Table III. Tumor related genes targeted by miRNAs from K562-derived MVs.

A, Oncogenes targeted by microRNAs in K562-derived MVs

\begin{tabular}{llll}
\hline Oncogene & \multicolumn{1}{c}{ microRNA species } & Oncogene & microRNA species \\
\hline BCL3 & miR-27blmiR-27a & RAB4B & miR-24 \\
BCL7A & miR-1202lmiR-21 & RAB5A & miR-494 \\
CASC4 & miR-575 & RAB8B & miR-23almiR-23b \\
CBL & miR-425 & RAP1A & miR-24 \\
ERBB3 & miR-22lmiR-24 & RAP2C & miR-93lmiR-24 \\
ERBB4 & miR-193bl miR-23almiR-23b & RAN & miR-134 \\
ETS1 & miR-1202 & RASL11B & miR-93 \\
FYN & miR-125a-3p & RASSF2 & miR-93 \\
MCL1 & miR-93 & RASD1 & miR-93 \\
MLF2 & miR-93 & RBBP4 & miR-26almiR-26b \\
MTUS1 & miR-361-5p & RHOU & miR-93 \\
MYCT1 & miR-23almiR-23b & RHOC & miR-23a,miR-23blmiR-223 \\
RAB11A & miR-21 & RRAS2 & miR-21 \\
RAB21 & miR-26almiR-26b & SKI & miR-193b \\
RAB35 & miR-185 & STMN1 & miR-26almiR-26b \\
RAB39B & miR-23almiR-23b & TET3 & \\
\hline
\end{tabular}

B, Tumor suppressors targeted by microRNAs in K562-derived MVs

\begin{tabular}{llll}
\hline Tumor suppressor & \multicolumn{1}{c}{ microRNA species } & Tumor suppressor & \multicolumn{1}{c}{ microRNA species } \\
\hline BAK1 & miR-26almiR-26blmiR-27blmiR-27a & NF1 & miR-103,miR-107lmiR-128I miR-125a-3p \\
BCR & miR-26almiR-26b & PHF6 & miR-26a \\
BRCA1 & miR-125a-3p & PTEN & miR-22lmiR-494lmiR-29a miR-29b \\
BRMS1L & miR-93 & RGS4 & miR-26almiR-26b \\
BTG3 & miR-93 & TP53INP1 & miR-22 \\
FOXO1 & miR-223 & TUSC2 & miR-23almiR-93lmiR-23b \\
IKZF4 & miR-575 & & \\
\hline
\end{tabular}

miR, microRNA; MV, microvesicle; BCL3, B-cell CLL/lymphoma 3; BCL7A, BCL tumor suppressor 7A; CASC4, cancer susceptibility candidate 4; CBL, Casitas B-lineage lymphoma; ERBB3, erb-b2 receptor tyrosine kinase 3; ERBB4, erb-b2 receptor tyrosine kinase 4; ETS1, E26 transformation specific sequence 1; FYN, Fibroblast Yes related novel; MCL1, myeloid cell leukemia sequence 1; MLF2, myeloid leukemia factor 2; MTUS1, microtubule associated tumor suppressor1; MYCT1, myc target 1; RAB11A, RAB21, RAB35 and RAB39Bare members RAS oncogene family; BAK1, BCL2 antagonist/killer 1; BCR, breakpoint cluster region; BRCA1; BRMS1L, breast cancer metastasissuppressor 1-like; BTG3, B cell translocation gene anti-proliferation factor 3; FOXO1, orkhead box O1; IKZF4, IKAROS family zinc finger 4.

and the results correlated well with the findings of microarray analysis.

Putative microRNA target genes analysis and functional annotation. To further study the functions of the aberrantly expressed microRNAs in MVs and in their parental cells, a predicted target analysis for these microRNAs was performed. The data revealed that 23 microRNAs exhibited altered expression in K562-derived MVs compared with control MVs. These microRNAs targeted 1,354 regulatory genes, which affect cellular apoptosis, proliferation and molecular signaling pathways. Notably, 43 oncogenes and tumor suppressor genes were identified among these aberrant microRNAs detected in MVs derived from K562 cells (Table III). In these dysregulated
microRNAs, 4 microRNAs targeted 2 oncogenes of the B-cell lymphoma family, while 12 microRNAs targeted 16 oncogenes of the rat sarcoma viral oncogene homolog (RAS) family. In addition, 24 microRNAs targeted 22 housekeeping genes in the p53 signaling pathway (Table IV). p53 is a tumor suppressor protein that regulates the expression of a wide variety of genes (34). It has been reported that the p53 signaling pathway has a key role in the induction of apoptosis of CML cells (35). With the exception of breast cancer 1 , which was targeted by miR-125a-3p, and insulin-like growth factor 1 , which was targeted by miR-1275 and miR-1207-5p, 16 of the above housekeeping genes were targeted by the downregulated microRNAs.

A predicted target analysis was also performed for the 104 microRNAs co-expressed in MVs and in their parental 
Table IV. Housekeeping genes in the p53 signaling pathway that are targeted by microRNAs in K562-derived microvesicles.

\begin{tabular}{|c|c|c|}
\hline MicroRNAs & Genes & Role in the p53 pathway \\
\hline $\operatorname{miR}-185$ & BAI1 & $\begin{array}{l}\text { Negative regulation of cell proliferation, } \\
\text { inhibition of angiogenesis and metastasis }\end{array}$ \\
\hline miR-26almiR-26blmiR-27blmiR-27a & BAK1 & Apoptosis \\
\hline miR-26almiR-26b & BID & Induction of apoptosis \\
\hline $\operatorname{miR}-125 a-3 p$ & BRCA1 & Apoptosis, DNA repair and cell proliferation \\
\hline $\operatorname{miR}-93$ & CASP2 & Anti-apoptosis \\
\hline $\operatorname{miR}-93$ & CCNG2 & Cell cycle checkpoint \\
\hline miR-23almiR-23b & $\mathrm{CCNH}$ & Cell cycle genes \\
\hline miR-27blmiR-27a & CHEK2 & Cell cycle arrest \\
\hline $\operatorname{miR}-22$ & CYR61 & Cell proliferation \\
\hline miR-1275lmiR-1207-5p & IGF1 & Anti-apoptosis \\
\hline miR-23alhsa-let-7flhsa-let-7elhsa-let-7c & FAS & Apoptosis \\
\hline $\begin{array}{l}\text { hsa-let-7blhsa-let-7almiR-23blhsa-let-7g } \\
\text { miR-21 }\end{array}$ & MSH2 & $\begin{array}{l}\text { DNA repair genes and negative regulation of the } \\
\text { cell cycle }\end{array}$ \\
\hline $\begin{array}{l}\text { miR-125a-3plmiR-103। } \\
\text { miR-107lmiR-128 }\end{array}$ & NF1 & $\begin{array}{l}\text { Negative regulation of cell proliferation and } \\
\text { negative regulation of the cell cycle }\end{array}$ \\
\hline miR-27blmiR-128lmiR-27a & PHB & $\begin{array}{l}\text { Associated with cell growth, proliferation and } \\
\text { differentiation }\end{array}$ \\
\hline miR-103ImiR-107 & RAI14 & Apoptosis \\
\hline miR-27blmiR-27a & SESN2 & Cell cycle arrest \\
\hline $\operatorname{miR}-223$ & SNCA & Anti-apoptosis \\
\hline miR-93 & MCL1 & Anti-apoptosis \\
\hline miR-24 & TSHR & Positive regulation of cell proliferation \\
\hline $\operatorname{miR}-24$ & PIGS & Apoptosis \\
\hline
\end{tabular}

miR, microRNA; hsa, Homo sapiens; BAI1, brain-specific angiogenesis inhibitor 1; BAK1, BCL2 antagonist/killer 1; BID, BH3 interacting domain death agonist; BRCA1, breast cancer 1, DNA repair associated; CASP2, caspase 2; CCNG2, cyclin G2; CCNH, cyclin H; CHEK2, checkpoint kinase 2; CYR61, cysteine rich angiogenic inducer 61; IGF1, insulin like growth factor 1; FAS, Fas cell surface death receptor; MSH2, mutS homolog 2; NF1, neurofibromin 1; PHB, prohibitin; RAI14, retinoic acid induced 14; SESN2, sestrin 2; SNCA, synuclein alpha; MCL1, myeloid cell leukemia sequence 1; TSHR, thyroid stimulating hormone receptor; PIGS, phosphatidylinositol glycan anchor biosynthesis class $\mathrm{S}$.

K562 cells. In total, 97 microRNAs targeted 3,743 regulatory genes. Data regarding miR-17*, miR-191*, miR-1914*, miR-1974_v14.0, miR-1977_v14.0, miR-1979_v15.0 and miR-33b* were not available in TargetScan 5.1.

Gene ontology (GO) analysis revealed that the highenrichment GOs targeted by the microRNAs co-expressed in MVs and the corresponding K562 cells were involved in various processes, including cell communication, signal transduction, RNA metabolism, transcription and cell differentiation. The pathway analysis revealed that there were 13 different pathways corresponding to the target genes, 12 of which were enriched $(\mathrm{P}<0.01)($ Table $\mathrm{V})$.

Mitogen-activated protein kinase (MAPK) signaling pathway is regulated by microRNAs in K562-MVs and K562 cells. To elucidate the mechanisms by which microRNAs co-expressed in MVs and parental K562 cells regulated leukemogenesis, a bioinformatic approach was undertaken to identify signaling pathways and target genes regulated by these microRNAs Using SAS v3.0, which is designed to integrate microRNA target genes into signaling pathways, mRNAs involved in the
MAPK signaling pathway, focal adhesion pathway, insulin signaling pathway and Wnt signaling pathway were observed to be significantly enriched in these microRNA target genes. These target genes were analyzed, and it was noticed that 34 microRNAs targeted 44 genes of the MAKP pathway, suggesting that the MAPK signaling pathway may play a significant role in MVs and their parental K562 cells.

The microRNA-gene interaction networks are represented in Fig. 4. As indicated in Fig. 4, the miR-30 family (with the exception of miR-30a and miR-30d) contained the most targeted mRNAs, with degrees ranging from 6 to 9, while miR-17, miR-16 and miR-15a/b occupied an important position in the MAPK pathway. These microRNAs may be important in the pathogenesis of CML. It was speculated that miR-17, miR-16 and miR-15a/b may be the key regulators of the MAPK signaling pathway in CML cells.

Chromosomal localization. A rearrangement (translocation) of genetic material between chromosomes 9 and 22 is associated with several types of blood cancer, particularly CML (36,37). As a typical CML cell line, K562 cells 
Table V. Target gene-associated pathways.

\begin{tabular}{lccc}
\hline Term & Number & q-value & Enrichment test P-value \\
\hline Cell cycle & 3 & 0.1089 & 0.9065 \\
Notch signaling pathway & 8 & 0.0014 & 0.0053 \\
Chronic myeloid leukemia & 18 & 0.0000 & 0.0000 \\
VEGF signaling pathway & 18 & 0.0000 & 0.0000 \\
mTOR signaling pathway & 23 & 0.0000 & 0.0000 \\
TGF $\beta$ signaling pathway & 23 & 0.0000 & 0.0000 \\
p53 signaling pathway & 24 & 0.0000 & 0.0000 \\
ErbB signaling pathway & 26 & 0.0000 & 0.0000 \\
Jak-STAT signaling pathway & 26 & 0.0000 & 0.0000 \\
Wnt signaling pathway & 41 & 0.0000 & 0.0000 \\
Insulin signaling pathway & 46 & 0.0000 & 0.0000 \\
Focal adhesion & 61 & 0.0000 & 0.0000 \\
MAPK signaling pathway & 90 & 0.0000 & 0.0000
\end{tabular}

VEGF, vascular endothelial growth factor; mTOR, mechanistic target of rapamycin; TGF, transforming growth factor; Jak, Janus kinase; STAT, signal transducer and activator of transcription; MAPK, mitogen-activated protein kinase.

Table VI. Target genes of microRNAs mapped to chromosomes 9 and 22 in K562 microvesicles and K562 cells.

\begin{tabular}{llcr}
\hline MicroRNAs & \multicolumn{1}{c}{ Targets $^{\text {a }}$} & -ln (P-value) & Pathways in CML \\
\hline hsa-miR-126 & CRK & 2.13 & 1 \\
miR-24 & CDKN1B, GAB2, ACVR1B, PIK3R3 & 1.17 & $1,2,3$ \\
miR-27b & TGFBR1, KRAS, CDK6, SOS1, RUNX1, & 4.26 \\
& GRB2, CBLB, ACVR1C, SHC4, PIK3CD & $1,2,4$ \\
hsa-let-7a,hsa-let-7b, & GAB2, TGFBR1, CDKN1A, CBL, E2F2, RB1, TP53, & 10.85 \\
hsa-let-7f & AKT2, ACVR1B, ACVR1C, BCL2L1, CCND & 9.80 \\
miR-7 & RB1, BCR, PIK3CD, PIK3R3, BCL2L1, RAF1 & 3.95 \\
miR-23b & STAT5B, SOS1, PTPN11, RUNX1, TGFBR2, & $1,2,3,4,5$ \\
miR-130b & ACVR1C, PIK3R3, CCND1 & 4.87 & $1,4,5$ \\
& TGFBR1, CDKN1A, SOS2, E2F2, MAPK1, TGFBR2, & 2
\end{tabular}

1, PI3K/Akt signaling pathway; 2 , TGF- $\beta$ signaling pathway; 3 , apoptosis; 4 , cell cycle; 5 , p53 signaling pathway; 6 , MAPK signaling. miR, microRNA; CML, chronic myeloid leukemia; hsa, Homo sapiens; CRK, v-crk avian sarcoma virus CT10 oncogene homolog; CDKN1B, cyclin dependent kinase inhibitor 1B; GAB2, GRB2 associated binding protein 2; ACVR1B, activin A receptor type 1B; PIK3R3, phosphoinositide3-kinase regulatory subunit 3; TGFBR1, transforming growth factor beta receptor 1; KRAS, Kirsten ras oncogene homolog; CDK6, cyclin dependent kinase 6; SOS1, SOS Ras/Rac guanine nucleotide exchange factor 1; RUNX1, runt related transcription factor 1; GRB2, growth factor receptor bound protein 2 ; CBLB, Cbl proto-oncogene B; ACVR1C, activin A receptor type 1C; SHC4, SHC adaptor protein 4; PIK3CD, phosphatidylinositol-4,5-bisphosphate 3-kinase catalytic subunit delta; CDKN1A, cyclin dependent kinase inhibitor 1A; CBL, Casitas B-lineage lymphoma; E2F2, E2F transcription factor 2; RB1, retinoblastoma 1; TP53, tumor protein p53; AKT2, AKT serine/threonine kinase 2; CCND1,cyclin D1; BCR, breakpoint cluster region; BCL2L1, BCL2 like 1; RAF1, v-raf-leukemia viral oncogene 1; STAT5B, signal transducer and activator of transcription 5B; PTPN11, protein tyrosine phosphatase, non-receptor type 11; TGFBR2, transforming growth factor beta receptor 2; SOS2, SOS Ras/Rho guanine nucleotide exchange factor 2; MAPK1, mitogen-activated protein kinase 1; SMAD4, SMAD family member 2 .

presented $t(9: 22)$ (38); thus, the chromosome location of the differentially expressed microRNAs was further examined. Several differentially regulated microRNAs in K562-derived MVs, including miR-27b, miR-24, miR-23b and miR-126*, were located in chromosome 9 . A total of 12 co-expressed microRNAs in MVs and their corresponding parental K562 cells [Homo sapiens (hsa)-let-7a, hsa-let-7f, miR-126,
miR-126*, miR-23b, miR-24, miR-27b and miR-7] were mapped to chromosome 9, whereas hsa-let-7b, miR-1249, miR-130b and miR-185 were mapped to chromosome 22. The genomic locations of a number of these microRNAs were distant from the ABL1 and BCR gene locus (Fig. 5). Using target prediction programmes, 875 predicted targets were observed to be regulated by these 12 microRNAs, 


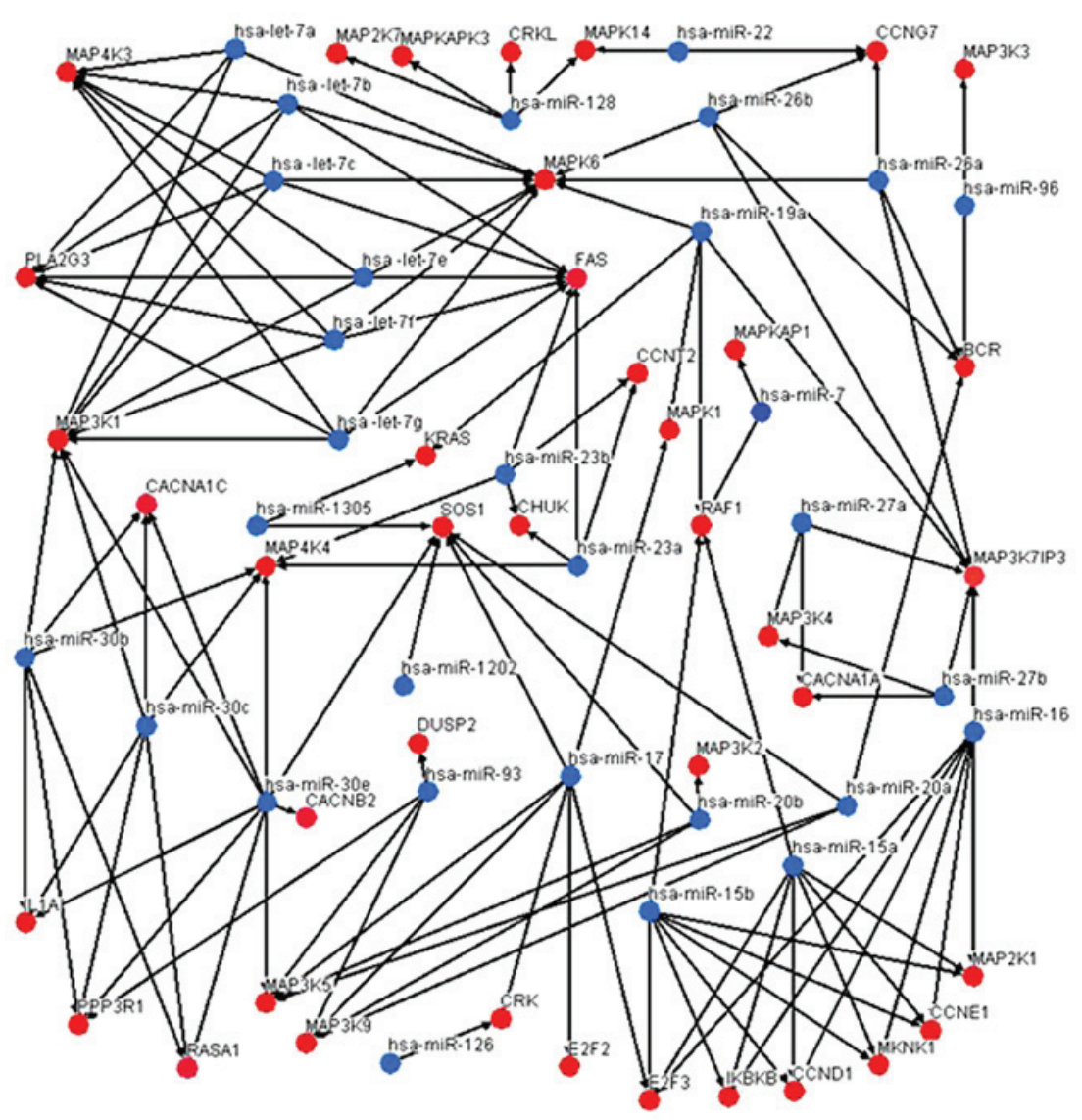

Figure 4. MicroRNA-gene interaction networks of the MAPK signaling pathway. Blue nodes represent microRNAs co-expressed in microvesicles derived from K562 cells and in K562 cells, while red nodes represent their target mRNAs. The edges represent the regulatory effect of microRNAs on mRNAs. miR-16, hsa-miR-17 and hsa-miR-15 family (miR-15a and miR-15b) were the most targeted mRNAs in the MAPK pathway (all exhibited a degree of 7). miRNA, microRNA; mRNA, messenger RNA; hsa, Homo sapiens; MAPK, mitogen-activated protein kinase; BCR, breakpoint cluster region; CCNT2, cyclin T2; CACNA1C, calcium voltage-gated channel subunit alpha1 C; CHUK, conserved helix-loop-helix ubiquitous kinase; CACNA1A, calcium voltage-gated channel subunit alpha1 A; CACNB2, calcium voltage-gated channel auxiliary subunit beta 2; CRK, v-crk avian sarcoma virus CT10 oncogene homolog; CCND1, cyclin D1; CCNE1, cyclin E1; CCNG7, cyclin G7; DUSP2, dual specificity phosphatase 2; E2F2, E2F transcription factor 2; E2F3, E2F transcription factor 3; FAS, Fas cell surface death receptor; IL1A, interleukin 1 alpha; IKBKB, inhibitor of kappa light polypeptide gene enhancer in B-cells, kinase beta; KRAS, Kirsten ras oncogene homolog; MAP4K3, mitogen-activated protein kinase kinase kinase kinase 3; MAP2K7, mitogen-activated protein kinase kinase 7; MAPKAPK3, mitogen-activated protein kinase-activated protein kinase 3; CRKL, CRK like proto-oncogene; MAPK14, mitogen-activated protein kinase 14; MAP3K3, mitogen-activated protein kinase kinase kinase 3; MAPK6, mitogen-activated protein kinase 6; MAPKAP1, mitogen-activated protein kinase associated protein 1; MAPK1, mitogen-activated protein kinase 1; MAP3K1, mitogen-activated protein kinase kinase kinase 1; MAP4K4, mitogen-activated protein kinase kinase kinase kinase 4; MAP3K7IP3, mitogen-activated protein kinase kinase kinase 7-interacting protein 3; MAP3K4, mitogen-activated protein kinase kinase kinase 4; MAP3K2, mitogen-activated protein kinase kinase kinase 2; MAP3K5, mitogen-activated protein kinase kinase kinase 5; MAP3K9, mitogen-activated protein kinase kinase kinase 9; MKNK1, MAP kinase interacting serine/threonine kinase 1; MAP2K1, mitogen-activated protein kinase kinase 1; PLA2G3, phospholipase A2 group III; PPP3R1, protein phosphatase 3 regulatory subunit B, alpha; RAF1, v-raf-leukemia viral oncogene 1; RASA1, RAS p21 protein activator 1; SOS1, SOS Ras/Rac guanine nucleotide exchange factor 1.

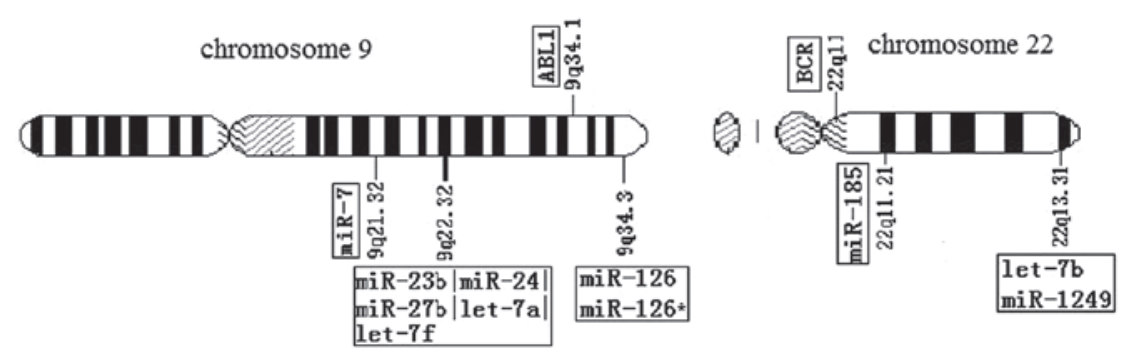

Figure 5. Map of chromosomes 9 and 22 indicating the locations of the microRNAs co-expressed in K562 cells and in K562 microvesicles. The genomic locations of these microRNAs were distant from the ABL1 and BCR gene locus. ABL1, Abelson murine leukemia viral oncogene homolog 1; BCR, breakpoint cluster region; miR, microRNA.

which may participate in leukemia via the above 875 genes. Using TargetScan 5.1 based on the KEGG pathway database, signaling pathways were analyzed for predicted targets involved in the MAPK, transforming growth factor $(\mathrm{TGF}) \beta$, p53, ErbB, Wnt, vascular endothelial growth factor, Notch and mechanistic target of rapamycin (mTOR) signaling 
pathways, focal adhesion and cell cycle. Table VI summarizes the predicted targets associated with CML.

Notably, it was observed that 70 of the 104 microRNAs co-expressed in MVs and in the corresponding parental K562 cells targeted 184 genes that were mapped to chromosome 9 , while 50 of the 104 microRNAs targeted 66 genes that were mapped to chromosome 22. These genes were correlated with the cell cycle, p53 signaling pathway, MAPK signaling pathway and RAS oncogene family.

\section{Discussion}

In the present study, K562 MVs microRNA expression was evaluated, and 7 microRNAs were identified to be significantly upregulated, while 16 were downregulated, in the K562-MV group compared with parental K562 cells (Table I). Those microRNAs may play a role in leukemia. MVs microRNAs may influence homeostasis (17). MVs in the leukemia microenvironment may target and fuse with normal cells, and transfer the dysfunctional microRNA to normal cells, consequently modifying normal cells in their environment to promote tumor growth, invasion and metastasis by various mechanisms.

The present study next assessed the microRNA expression profile of K562 cells. Compared with the microRNA expression profile of K562-derived MVs and their parental cells, 181/888 microRNAs $(20 \%)$ were present at elevated levels within MVs and 226/888 microRNAs (25\%) were present at a higher proportion in the parental cells. In addition, 104 microRNAs were co-expressed both in MVs and parental cells, which indicated that MVs microRNA content may, at least in part, reflect the expression of their parental cells. In other words, the microRNA expression profile of leukemia-derived MVs is a small version of that exhibited by the leukemia parental cells.

Notably, 104 co-expressed microRNAs in MVs and their parental cells included certain microRNA clusters and microRNA families associated with cancer. For example, the microRNA-23-27-24 cluster is associated with angiogenesis (39), and the oncomir miR-17-92 cluster (miR-17, miR-19a, miR-19b, miR-20a and miR-92a), which was detected in CML cluster of differentiation $34^{+}$cells (24), was also observed in the present study. These clusters encoded seven microRNAs that regulate cell proliferation, apoptosis and development (40).

Since microRNAs have a global effect on gene expression, it is not surprising that they may modulate leukemia progression. In the present study, a number of oncogenes and tumor suppressor genes were regulated by microRNAs. According to computational predictions, a single microRNA can target dozens of genes, and different microRNAs can target one gene (41). The microRNAs displayed co-expression both in $\mathrm{K} 562 \mathrm{MVs}$ and in the corresponding parental cells, indicating a common role in leukemia genesis or progression.

It was also observed that 122 elevated microRNAs were only expressed in parental cells, suggesting that the compartmentalization of microRNAs from cells into MVs, at least for certain microRNAs, is an active (selective) process.

The present study also identified target genes regulated by microRNAs that were dysregulated in MVs and in their parental K562 cells. It was observed that oncogenes and tumor suppressor genes were significantly enriched in these target genes. A large number of the genes identified in the present study as the potential targets of differentially regulated microRNAs are known to be involved in cancer through their effects on cell differentiation [cyclin-dependent kinase 6 and leukemia inhibitory factor receptor (LIFR)], apoptosis (proviral integration site 1) or hematopoiesis (GATA binding protein 2 and T-cell acute lymphocytic leukemia 1). Notably, numerous targets of the cancer-associated microRNAs, including activin A receptor (ACVR)1B, ACVR1C, Casitas B-lineage lymphoma $(\mathrm{CBL})$, cyclin $(\mathrm{CCN}) \mathrm{D} 1$, cyclin-dependent kinase inhibitor 1B,CT10 regulator of kinase (CRK), cold shock domain containing E1, ecotropic virus integration site 1 protein homolog, growth factor receptor-bound protein 2, histone deacetylase 2, LIFR, phosphoinositide-3-kinase regulatory subunit 3, phosphatidylinositol-4,5-bisphosphate 3-kinase catalytic subunit delta, RAF1, retinoblastoma 1, runt related transcription factor 1 , son of sevenless homolog 1 (SOS1), signal transducer and activator of transcription (STAT) $5 \mathrm{~B}, \mathrm{TGF} \beta$ receptor (TGFBR)2, TGFBR1 and tumor protein 53, have been previously reported to be associated with leukemogenesis $(42,43)$. K562 cells released MVs enriched in aberrantly expressed microRNAs, including oncomicroRNAs and tumor suppressor microRNAs, into the leukemia cells microenvironment, which may play a role in leukemia by abnormally regulating their target genes, including oncogenes and tumor suppressors, consequently resulting in leukemia.

The present study also identified signaling pathways regulated by microRNAs that were dysregulated in MVs and in their parental K562 cells. A large number of these target genes were involved in the MAPK, RAS, p53, ErbB, Janus kinase-STAT, TGF $\beta$, mTOR and Wnt signaling pathways, which are known to be associated with leukemia (44-50). Consistent with other cancers (51-53), the role of leukemia-derived MVs was to modulate the disease progression rather than being the main cause of the disease itself. MVs from leukemia cells facilitate leukemia progression and invasion in different ways (15,54-56). MVs are considered to be taken up by cells through endocytosis and to release their intravesicular content upon fusing their membrane with the endosomal membrane (52). Although the precise mechanisms of uptake are poorly understood, it is evident that release of microRNAs from the lumen of MVs can induce activation of specific signal transduction cascades and influence the physiologic state of recipient cells (52). MVs have an additional advantage over naturally secreted signaling molecules in that they can present multiple epitopes to the recipient cell, enabling co-stimulatory pathways to be activated (57).

Of the predicted targets of microRNAs co-expressed in MVs and their corresponding parental K562 cells, 30 were involved in MAPK signaling (including BCR, CBL, CCNE1, CCND1, CCNT2, CRK, CRK-like, E2F transcription factor (E2F)2, E2F3, Kirsten RAS, MAPK1, MAPK12, RAF1 and SOS1). The MAPK signaling pathway is a common point of convergence of various different mitogenic and anti-apoptotic signal transduction pathways in hematopoietic and epithelial cancer cells (45). Such deregulation of the MAPK pathway contributes to BCR-ABL leukemogenesis (58). The MVs derived from leukemia cells may weaken or enhance the expression of mRNAs involved in the MAPK pathway in recipient cells, and contribute to the development of leukemia. 
As K562 cells presented $t(9: 22)$, several differentially regulated microRNAs of K562 MVs and their parental K562 cells were investigated. For example, miR-27b, miR-24, miR-23b and miR-126* are known to be encoded by the chromosome 9 (59-61). In the present study, a considerable number of co-expressed microRNAs in MVs and K562 cells (including hsa-let-7a, hsa-let-7f, miR-126, miR-126", miR-23b, miR-24, miR-27b and miR-7) were mapped to chromosome 9, while let-7b, miR-1249, miR-130b and miR-185 were mapped to chromosome 22. The genomic locations of various of these microRNAs were distant from the ABL1 and BCR gene locus (Fig. 5). Alterations in chromosome 9 are associated with a large number of diseases, particularly cancer (62). Therefore, there may be a link between alterations in chromosome 9 and differential expression of microRNAs.

In summary, the present study first identified the microRNA expression profile of MVs derived from K562 cells and that of their parental K562 cells. The fact that 104 microRNAs were observed to be co-expressed both in MVs and their parental cells suggests that MVs microRNAs may, at least in part, reflect the microRNA expression profile of the parental cells. There were numerous oncogenes, tumour suppressors and signaling pathway genes that were targeted by these aberrantly expressed MVs microRNAs, which may contribute to the development of hematopoietic malignancies. Therefore, leukemia MVs microRNAs may represent a novel way to intervene therapeutically for treating CML.

\section{Acknowledgements}

The present study was supported by the National Natural Science Foundation of China (Beijing, China; grant no. 81170462). The authors would like to thank the staff at the Center for Stem Cell Research and Application (Institute of Hematology, Union Hospital, Tongji Medical College, Huazhong University of Science and Technology) for their technical assistance and the staff at the Clinical Laboratory of the Department of Infection Diseases (Institute of Hematology, Union Hospital, Tongji Medical College, Huazhong University of Science and Technology) for their help collecting samples.

\section{References}

1. Zhou JB, Zhang T, Wang BF, Gao HZ and Xu X: Identification of a novel gene fusion RNF213SLC26A11 in chronic myeloid leukemia by RNA-Seq. Mol Med Rep 7: 591-597, 2013.

2. Apperley JF: Chronic myeloid leukaemia. Lancet 385: 1447-1459, 2015.

3. Sasaki K and Mitani K: Molecular pathogenesis of chronic myeloid leukemia. Nihon Rinsho 67: 1894-1899, 2009 (In Japanese).

4. Belting $\mathrm{M}$ and Christianson HC: Role of exosomes and microvesicles in hypoxia-associated tumour development and cardiovascular disease. J Intern Med 278: 251-263, 2015.

5. Lo Cicero A, Schiera G, Proia P Saladino P, Savettieri G, Di Liegro $\mathrm{CM}$ and Di Liegro I: Oligodendroglioma cells shed microvesicles which contain TRAIL as well as molecular chaperones and induce cell death in astrocytes. Int J Oncol 39: 1353-1357, 2011.

6. Lee TH, D'Asti E, Magnus N, Al-Nedawi K, Meehan B and Rak J: Microvesicles as mediators of intercellular communication in cancer-the emerging science of cellular 'debris'. Semin Immunopathol 33: 455-467, 2011.

7. Pilzer D, Gasser O, Moskovich O, Schifferli JA and Fishelson Z: Emission of membrane vesicles: Roles in complement resistance, immunity and cancer. Springer Semin Immunopathol 27: 375-387, 2005 .
8. EL Andaloussi S, Mäger I, Breakefield XO and Wood MJ: Extracellular vesicles: Biology and emerging therapeutic opportunities. Nat Rev Drug Discov 12: 347-357, 2013.

9. Al-Nedawi K, Meehan B, Micallef J, Lhotak V, May L, Guha A and Rak J: Intercellular transfer of the oncogenic receptor EGFRvIII by microvesicles derived from tumour cells. Nat Cell Biol 10: 619-624, 2008.

10. Skog J, Würdinger T, van Rijn S, Meijer DH, Gainche L, Sena-Esteves M, Curry WT Jr, Carter BS, Krichevsky AM and Breakefield XO: Glioblastoma microvesicles transport RNA and proteins that promote tumour growth and provide diagnostic biomarkers. Nat Cell Biol 10: 1470-1476, 2008.

11. Pisetsky DS, Gauley J and Ullal AJ: Microparticles as a source of extracellular DNA. Immunol Res 49: 227-234, 2011.

12. Valadi H, Ekström K, Bossios A, Sjöstrand M, Lee JJ and Lötvall JO: Exosome-mediated transfer of mRNAs and microRNAs is a novel mechanism of genetic exchange between cells. Nat Cell Biol 9: 654-659, 2007.

13. Taverna S, Flugy A, Saieva L, Kohn EC, Santoro A, Meraviglia S, De Leo G and Alessandro R: Role of exosomes released by chronic myelogenous leukemia cells in angiogenesis. Int J Cancer 130: 2033-2043, 2012.

14. Mineo M, Garfield SH, Taverna S, Flugy A, De Leo G, Alessandro R and Kohn EC: Exosomes released by K562 chronic myeloid leukemia cells promote angiogenesis in a Src-dependent fashion. Angiogenesis 15: 33-45, 2012.

15. Szczepanski MJ, Szajnik M, Welsh A, Whiteside TL and Boyiadzis M: Blast-derived microvesicles in sera from patients with acute myeloid leukemia suppress natural killer cell function via membrane-associated transforming growth factor-beta1. Haematologica 96: 1302-1309, 2011.

16. Hunter MP, Ismail N, Zhang X, Aguda BD, Lee EJ, Yu L, Xiao T, Schafer J, Lee ML, Schmittgen TD, et al: Detection of microRNA expression in human peripheral blood microvesicles. PLoS One 3: e3694, 2008.

17. Taylor DD and Gercel-Taylor C: MicroRNA signatures of tumor-derived exosomes as diagnostic biomarkers of ovarian cancer. Gynecol Oncol 110: 13-21, 2008.

18. Wysoczynski M and Ratajczak MZ: Lung cancer secreted microvesicles: Underappreciated modulators of microenvironment in expanding tumors. Int J Cancer 125: 1595-1603, 2009.

19. Hayes J, Peruzzi PP and Lawler S: MicroRNAs in cancer: Biomarkers, functions and therapy. Trends Mol Med 20: 460-469, 2014.

20. Lu J, Getz G, Miska EA, Alvarez-Saavedra E, Lamb J, Peck D, Sweet-Cordero A, Ebert BL, Mak RH, Ferrando AA, et al: MicroRNA expression profiles classify human cancers. Nature 435: 834-838, 2005.

21. Volinia S, Galasso M, Costinean S, Tagliavini L, Gamberoni G, Drusco A, Marchesini J, Mascellani N, Sana ME, Abu Jarour R, et al: Reprogramming of miRNA networks in cancer and leukemia. Genome Res 20: 589-599, 2010.

22. Ramkissoon SH, Mainwaring LA, Ogasawara Y, Keyvanfar K, McCoy JP Jr, Sloand EM, Kajigaya S and Young NS: Hematopoietic-specific microRNA expression in human cells. Leuk Res 30: 643-647, 2006.

23. Venturini L, Battmer K, Castoldi M, Schultheis B, Hochhaus A, Muckenthaler MU, Ganser A, Eder M and Scherr M: Expression of the miR-17-92 polycistron in chronic myeloid leukemia (CML) CD34+ cells. Blood 109: 4399-4405, 2007.

24. Taylor DD and Gerçel-Taylor C: Tumour-derived exosomes and their role in cancer-associated T-cell signalling defects. Br J Cancer 92: 305-311, 2005.

25. Kosaka N, Iguchi H, Yoshioka Y, Takeshita F, Matsuki Y and Ochiya T: Secretory mechanisms and intercellular transfer of MicroRNAs in living Cells. J Biol Chem 285: 17442-17452, 2010.

26. Chen CZ, Li L, Lodish HF and Bartel DP: MicroRNAs modulate hematopoietic lineage differentiation. Science 303: 83-86, 2004.

27. Li T, Lu YY and Zhao XD, Guo HQ, Liu CH, Li H, Zhou L, Han YN, Wu KC, Nie YZ, et al: MicroRNA-296-5p increases proliferation in gastric cancer through repression of Caudalrelated homeobox 1. Onocgene 33: 783-793, 2014.

28. Li WY, Chen XM, Xiong W, Guo DM, Lu L and Li HY: Detection of microvesicle miRNA expression in ALL subtypes and analysis of their functional roles. J Huazhong Univ Sci Technolog Med Sci 34: 640-645, 2014.

29. Sun L, Hu J, Xiong W, Chen X, Li H and Jie S: MicroRNA expression profiles of circulating microvesicles in hepatocellular carcinoma. Acta Gastroenterol Belg 76: 386-392, 2013. 
30. Zhang L, Valencia C, Dong B, Chen M, Guan P and Pan L: Transfer of microRNAs by extracellular membrane microvesicles: A nascent crosstalk model in tumor pathogenesis, especially tumor cell-microenvironment interactions. J Hematol Oncol 8: 14, 2015

31. Giusti I, D'Ascenzo S and Dolo V: Microvesicles as potential ovarian cancer biomarkers. Biomed Res Int 2013: 703048, 2013.

32. Nieuwland R, Berckmans RJ, McGregor S, Böing AN, Romijn FP, Westendorp RG, Hack CE and Sturk A: Cellular origin and procoagulant properties of microparticles in meningococcal sepsis. Blood 95: 930-935, 2000

33. Vaz C, Ahmad HM, Sharma P, Gupta R, Kumar L, Kulshreshtha R and Bhattacharya A: Analysis of microRNA transcriptome by deep sequencing of small RNA libraries of peripheral blood BMC Genomics 11: 288, 2010

34. Linderholm B, Norberg T and Bergh J: Sequencing of the tumor suppressor gene TP 53. Methods Mol Med 120: 389-401, 2006.

35. Di Bacco A, Keeshan K, McKenna SL and Cotter TG: Molecular abnormalities in chronic myeloid leukemia: Deregulation of cell growth and apoptosis. Oncologist 5: 405-415, 2000.

36. Gilbert F: Disease genes and chromosomes: Disease maps of the human genome. Chromosome 22. Genet Test 2: 89-97, 1998.

37. Gilbert F and Kauff N: Disease genes and chromosomes: Disease maps of the human genome. Chromosome 9. Genet Test 5: 157-174, 2001.

38. Chai JH, Zhang Y, Tan WH, Chng WJ, Li B and Wang X: Regulation of hTERT by BCR-ABL at multiple levels in K562 cells BMC Cancer 11: 512, 2011.

39. Zhou Q, Gallagher R, Ufret-Vincenty R, Li X, Olson EN and Wang S: Regulation of angiogenesis and choroidal neovascularization by members of microRNA-23 27 24 clusters. Proc Natl Acad Sci USA 108: 8287-8292, 2011.

40. Pospisil V, Vargova K, Kokavec J, Rybarova J, Savvulidi F, Jonasova A, Necas E, Zavadil J, Laslo P and Stopka T: Epigenetic silencing of the oncogenic miR-17-92 cluster during PU.1-directed macrophage differentiation. EMBO J 30: 4450-4464, 2011.

41. Lawrie CH, Chi J, Taylor S, Tramonti D, Ballabio E, Palazzo S, Saunders NJ, Pezzella F, Boultwood J, Wainscoat JS and Hatton CS: Expression of microRNAs in diffuse large B cell lymphoma is associated with immunophenotype, survival and transformation from follicular lymphoma. J Cell Mol Med 13: 1248-1260, 2009.

42. Zhao H, Wang D, Du W, Gu D and Yang R: MicroRNA and leukemia: Tiny molecule, great function. Crit Rev Oncol Hematol 74: 149-155, 2010.

43. Cammarata G, Augugliaro L and Salemi D, Agueli C, La Rosa M, Dagnino L, Civiletto G, Messana F, Marfia A, Bica MG, et al: Differential expression of specific microRNA and their targets in acute myeloid leukemia. Am J Hematol 85: 331-339, 2010.

44. Zenz T, Mohr J and Edelmann J, Sarno A, Hoth P, Heuberger M, Helfrich H, Mertens D, Dohner H, Stilgenbauer S: Treatment resistance in chronic lymphocytic leukemia: The role of the p53 pathway. Leuk Lymphoma 50: 510-513, 2009.

45. Wang X, Pesakhov S and Weng A, Kafka M, Gocek E, Nguyen M, Harrison JS, Danilenko M, Studzinski GP: ERK 5/MAPK pathway has a major role in $1 \alpha, 25-(\mathrm{OH}) 2$ vitamin $\mathrm{D} 3$-induced terminal differentiation of myeloid leukemia cells. J Steroid Biochem Mol Biol 144: 223-227, 2014.
46. Caye A, Strullu M and Guidez F, Cassinat B, Gazal S, Fenneteau O, Lainey E, Nouri K, Nakhaei-Rad S, Dvorsky R, et al: Juvenile myelomonocytic leukemia displays mutations in components of the RAS pathway and the PRC2 network. Nat Genet 47: 1334-1340, 2015.

47. Ufkin ML, Peterson S, Yang X, Driscoll H, Duarte C and Sathyanarayana P: miR-125a regulates cell cycle, proliferation, and apoptosis by targeting the ErbB pathway in acute myeloid leukemia. Leuk Res 38: 402-410, 2014

48. Hornakova T, Staerk J and Royer Y, Flex E, Tartaglia M, Constantinescu SN, Knoops L, Renauld JC: Acute lymphoblastic leukemia-associated JAK1 mutants activate the Janus kinase/STAT pathway via interleukin-9 receptor alpha homodimers. J Biol Chem 284: 6773-6781, 2009.

49. Dinner S and Platanias LC: Targeting the mTOR pathway in Leukemia. J Cell Biochem 117: 1745-1752, 2016.

50. Eaves CJ and Humphries RK: Acute myeloid leukemia and the Wnt pathway. N Engl J Med 362: 2326-2327, 2010.

51. Arshad Malik MF: Influence of microvesicles in breast cancer metastasis and their therapeutic implications. Arch Iran Med 18: 189-192, 2015.

52. Antonyak MA and Cerione RA: Microvesicles as mediators of intercellular communication in cancer. Methods Mol Biol 1165: $147-173,2014$

53. Jorfi $\mathrm{S}$ and Inal JM: The role of microvesicles in cancer progression and drug resistance. Biochem Soc Trans 41: 293-298, 2013.

54. Wang Y, Cheng Q, Liu J and Dong M: Leukemia Stem CellReleased Microvesicles Promote the Survival and Migration of Myeloid Leukemia Cells and These Effects Can Be Inhibited by MicroRNA34a Overexpression. Stem Cell Int 2016: 9313425, 2016.

55. Lu L, Chen XM, Tao HM, Xiong W, Jie SH and Li HY: Regulation of the expression of zinc finger protein genes by microRNAs enriched within acute lymphoblastic leukemia-derived microvesicles. Genet Mol Res 14: 11884-11895, 2015.

56. Ghosh AK, Secreto CR, Knox TR, Ding W, Mukhopadhyay D and Kay NE: Circulating microvesicles in B-cell chronic lymphocytic leukemia can stimulate marrow stromal cells: Implications for disease progression. Blood 115: 1755-1764, 2010.

57. van der Vos KE, Balaj L, Skog J and Breakefield XO: Brain tumor microvesicles: insights into intercellular communication in the nervous system. Cell Mol Neurobiol 31: 949-959, 2011.

58. Redig AJ, Vakana E and Platanias LC: Regulation of mammalian target of rapamycin and mitogen activated protein kinase pathways by BCR-ABL. Leuk Lymphoma 52 (Suppl 1): S45-S53, 2011.

59. Liang T, Yu J, Liu C and Guo L: An exploration of evolution, maturation, expression and function relationships in mir-23 27 24 cluster. PLoS One 9: e106223, 2014.

60. Yan J, Ma S, Zhang Y, Yin C, Zhou X and Zhang G: Potential role of microRNA-126 in the diagnosis of cancers: A systematic review and meta-analysis. Medicine (Baltimore) 95: e4644, 2016.

61. Xiao J, Lin HY, Zhu YY, Zhu YP and Chen LW: MiR-126 regulates proliferation and invasion in the bladder cancer BLS cell line by targeting the PIK3R2-mediated PI3K/Akt signaling pathway. Onco Targets Ther 9: 5181-5193, 2016

62. Grady B, Goharderakhshan R, Chang J, Ribeiro-Filho LA, Perinchery G, Franks J, Presti J, Carroll P and Dahiya R: Frequently deleted loci on chromosome 9 may harbor several tumor suppressor genes in human renal cell carcinoma. J Urol 166: 1088-1092, 2001. 